\title{
Révolution française et littérature anglaise
}

\author{
Matthew 0. Grenby
}

\section{OpenEdition \\ Journals}

Édition électronique

URL : https://journals.openedition.org/ahrf/1919

DOI : 10.4000/ahrf.1919

ISSN : 1952-403X

Éditeur :

Armand Colin, Société des études robespierristes

Édition imprimée

Date de publication : 1 décembre 2005

Pagination : 101-144

ISSN : 0003-4436

Référence électronique

Matthew O. Grenby, «Révolution française et littérature anglaise ». Annales historiques de la Révolution française [En ligne], 342 | octobre-décembre 2005, mis en ligne le 15 décembre 2008, consulté le 22 avril 2022. URL : http://journals.openedition.org/ahrf/1919 ; DOI : https://doi.org/10.4000/ahrf.1919

Ce document a été généré automatiquement le 22 avril 2022.

Tous droits réservés 


\title{
Révolution française et littérature anglaise
}

\author{
Matthew 0. Grenby
}

1 Reconnaitre que la Révolution française a eu un impact considérable sur la littérature anglaise n'est pas nouveau. Déjà en 1818, William Hazlitt soutenait que toute «l'école lakiste » de la poésie anglaise "trouvait son origine dans la Révolution française, ou plutôt dans les sentiments et opinions qui provoquèrent cette révolution " ${ }^{1}$. Ceci n'était pas non plus une grande révélation. Les poètes eux-mêmes admettaient ouvertement cette influence. Shelley écrivit que le génie des romantiques anglais tenait «moins à leur propre esprit qu'à l'esprit de l'époque " ${ }^{2}$. De fait, on ne peut passer sous silence les liens qui existent entre la Révolution française et la créativité littéraire britannique. Presque tous les écrivains de premier plan des années 1790 - de Burke à Burns, de Wollstonecraft à Wordsworth - s'exprimèrent volontiers sur les événements en France et sur leurs réactions inconstantes envers une Révolution elle-même changeante. Le sens symbolique des événements en France domina toute l'œuvre de Blake après 1789 , qui comprend un poème d'une importance majeure au titre sans fioritures: The French Revolution (1791). Les mots de Wordsworth: «Le bonheur, en cette aube, était de vivre", et France: An Ode (1798) de Coleridge ne sont que les exemples les plus pertinents et les plus connus ${ }^{3}$. L'influence de la Révolution française sur la littérature anglaise ne diminua pas non plus à cause de Napoléon, voire de Waterloo. Plus tard, les romantiques étaient heureux d'avouer qu'ils continuaient à subir l'influence de ce que De Quincey appela la «grande tempête morale» de la Révolution ${ }^{4}$. Par la suite, ces réactions rejoignirent sans aucune démarcation ce qu'exprimaient les écrits des générations ultérieures à propos des événements qui s'étaient produits en France de nombreuses années auparavant, tels The French Revolution (Histoire de la Révolution française) (1837) de Carlyle, ou A Tale of Two Cities (Un conte de deux villes) (1859) de Dickens. Pendant plus ou moins un siècle après son début, la Révolution resta au cœur de la littérature anglaise.

2 Cette relation entre la créativité britannique et la révolte en France n'avait pas échappé aux critiques littéraires. En fait, appréhender le romantisme britannique 
comme un mouvement cohérent plutôt que comme un rassemblement d'écrivains individuels qui travaillaient séparément, est dû à la perception que les spécialistes de littérature eurent de l'impact de la Révolution française. Toutes les citations du paragraphe ci-dessus furent utilisées par M.H. Abrams à l'appui de ce qu'il avançait dans son essai de la plus haute importance intitulé English Romanticism: The Spirit of the Age (1963). D'après Abrams, ce fut la Révolution française qui créa le romantisme anglais, plus particulièrement les espoirs contrariés des poètes britanniques que la Révolution aurait fait entrer dans une ère nouvelle de liberté et de Lumières. Lorsque la Révolution devint répressive, ainsi que l'indique Abrams, l'idéalisme des poètes fut intériorisé, et un nouveau mouvement littéraire égalitariste, débordant d'énergie et d'imagination vit le jour ${ }^{5}$. Ainsi que nous le verrons, tout le monde n'accepte pas, à l'heure actuelle, cette explication plutôt habile de la créativité des romantiques, pas plus que la manière dont Abrams la réserve exclusivement à quelques auteurs de sexe masculin. Les générations de critiques qui ont suivi n'ont cependant pas tenté de rompre le lien entre la Révolution et les écrits britanniques. Loin de là. Au lieu de cela, ils ont consacré leur temps à une analyse plus fouillée pour déterminer ce que « l'esprit de l'époque » était, et à l'étude des relations entre texte et contexte, pour tous les écrivains, et pas seulement pour ceux qui, explicitement, écrivaient sur des questions politiques.

3 De fait, ce que cette courte étude montrera est l'effet spectaculaire de ce que l'on appelle le "nouvel historicisme» sur les recherches concernant l'impact de la Révolution française. Les changements les plus nets sont la pluralité des travaux récents sur les réactions des Britanniques à la Révolution française; le nombre des écrivains dont l'œuvre est à l'heure actuelle examinée minutieusement, et l'abolition de la distinction auparavant faite entre personnalités « de premier » et « de second plan » sont remarquables. Ainsi, désormais, il est davantage tenu compte des écrits de femmes, des écrivains noirs, de la littérature enfantine, des écrits en provenance de différentes régions, de textes pour des publics différents, des journaux intimes, des articles de presse, des guides de bonne conduite, et de presque toute autre forme de production littéraire. Il y a également davantage de recherches sur les lecteurs intéressés par ces différents types d'œuvres et le rôle qu'ils ont joué dans leur élaboration. Un second changement évident réside dans la manière dont les chercheurs, lorsqu'ils écrivent sur la Révolution française et la littérature britannique, ne pensent plus seulement en termes de lien direct de cause à effet entre les événements historiques et la production culturelle, mais considèrent maintenant que le texte et le contexte sont étroitement liés à tous les niveaux, et s'enrichissent l'un l'autre à tout moment. Ceci signifie que les travaux les plus intéressants dont nous disposons à l'heure actuelle sur cette période ne sont plus de simples études sur l'impact de la Révolution française sur la littérature britannique; ils portent plutôt sur des questions plus vastes qui font intervenir la Révolution française et le sentiment de crise qui en résulta en tant qu'éléments importants du contexte. Dans de tels travaux, la Révolution française n'est généralement pas considérée comme un événement qui a tout transfiguré et brusquement tout bouleversé en 1789 ou en 1793. Plus exactement, des changements importants d'ordre politique, économique, social et culturel étaient déjà en cours et se poursuivirent pendant les années 1790 à 1800 . De même, les chercheurs à l'heure actuelle ne tendent pas à voir la Révolution comme une série d'événements qui se produisirent en France et que les Britanniques perçurent ensuite avec passivité, qu'ils discutèrent et auxquels ils réagirent. C'est plutôt le sentiment 
d'une crise qui se manifesta en Grande-Bretagne, et non les événements en France par eux-mêmes, que les chercheurs en littérature considèrent souvent, aujourd'hui, comme étant la «Révolution française ". Les écrivains réagirent à cette crise, et leurs textes influencèrent son déroulement, ainsi que la manière dont les événements de l'autre côté de la Manche furent appréhendés. La réalité de la Révolution en France n'est pas devenue moins importante pour les chercheurs anglo-américains, mais ils en sont venus à la considérer comme faisant partie d'une histoire plus vaste dans laquelle processus et discours sont intimement liés.

4 Gerald Izenberg dans Impossible Individuality: Romanticism, Revolution and the Origins of Modern Selfhood, 1787-1802 donne un bon exemple d'une telle observation. Ainsi que le suggère le titre, ce livre traite de la construction de notre concept moderne d'individualité, en considérant Wordsworth, Schegel et Chateaubriand entre autres. Ces changements étaient déjà en cours, d'après Izenberg, mais la Révolution fut cependant d'une importance capitale, telle l'enclume sur laquelle la perception moderne de l'individualité était forgée. The Domestic Revolution : Enlightenment Feminisms and the Novel d'Eve Tavor Bannet et The Culture of Sensibility: Sex and Society in Eighteenth-Century Britain de G.J. Barker-Benfield sont deux exemples supplémentaires. Ces ouvrages étudient des aspects de la culture britannique tout au long du XVIII ${ }^{\mathrm{e}}$ siècle, mais tous deux soulignent également que la crise de la Révolution française des années 1790 fut cruciale dans la redéfinition de ce qui était possible et convenable pour une femme. Pour Barker-Benfield, la Révolution française conduisit Mary Wollstonecraft, Hannah More et d'autres à réécrire le code de la sensibilité qui avait, pendant des décennies, été utilisé pour définir les femmes et leur imposer des limites. Dans l'ouvrage de Bannet, l'importance de la Révolution française elle-même est en fait minimisée, si on la compare par exemple, avec les changements dans les comportements et les attitudes que les auteurs britanniques firent lentement naître tout au long du XVIII ${ }^{e}$ siècle. Mais Bannet indique que les réactions britanniques à la Révolution française restèrent cependant primordiales pour la formulation et la re-formulation de l'idée de la vie de famille. Dans de tels livres, la crise de la Révolution n'est pas la seule "grande cause " de nouvelles formes de production littéraire, mais trouve sa place dans des récits historiographiques plus complexes, et elle n'est comprise qu'à la lumière de textes antérieurs, aussi bien que de ceux écrits après coup. Comme tant d'autres textes récents de la " nouvelle historicité » examinés dans le cadre de cet article, les ouvrages d'Izenberg, de Barker-Benfield et de Bannet utilisent des textes pour expliquer des contextes, aussi bien que des contextes pour expliquer des textes.

5 Les ouvrages de ce genre ont été si nombreux au cours des vingt dernières années qu'il ne sera pas possible de tenir compte de tous dans cette présente étude. Afin de tenter d'imposer un ordre dans la profusion des documents qui ont été publiés dans ce domaine, je diviserai le reste de mon propos en quelques catégories de base selon le genre considéré : poésie, théâtre, roman, et faute d'une expression plus satisfaisante, écrits divers. Il existe cependant de nombreuses études qui ne tiennent pas compte de ces distinctions. Parmi les ouvrages importants qui portent sur différents genres et ont jalonné le terrain se trouvent Romantics, Rebels and Reactionaries de Marilyn Butler, qui, le premier, appela à un examen nuancé du lien entre créativité romantique et processus historique, The French Revolution and the Enlightnment in England de Seamus Deane, qui dresse un état de l'influence des écrivains français du Siècle des Lumières sur les révolutionnaires britanniques, et, plus récemment, The Crisis of Literature in the 1790s de Paul Keen qui montre que les appréhensions politiques en Grande-Bretagne 
après la Révolution cristallisèrent des inquiétudes réelles concernant la mobilité sociale et un lectorat plus étendu, et eurent pour conséquence un réexamen de la place véritable de la littérature dans la société ${ }^{6}$. Bien qu'elles traitent d'aspects plus spécifiques de l'impact des événements en France sur la littérature britannique, on relève d'autres études générales; ainsi: Napoleon and English Romanticism de Simon Bainbridge, Romanticism and War: A Study of the British Romantic Writers and the Napoleonic Wars de J.R. Watson, l'article d'Anne Mellor «English Women Writers and the French Revolution » et enfin l'ouvrage à venir d'Adriana Craciun British Women Writers and the French Revolution.

6 Tout au long de la discussion qui suit, on remarquera d'emblée deux points. Le premier, sans doute produit par de la «désacralisation» des études littéraires et la quête de nouvelles voies, est que -alors que les études plus anciennes de la Révolution française et de la littérature britannique avaient tendance à s'occuper surtout de la poésie des "grands" romantiques, en particulier Blake, Wordsworth et Coleridge c'est la prose qui a attiré davantage l'attention au cours des dernières années. Le deuxième est que la Révolution française donna de toute évidence naissance à une crise sérieuse d'introspection en Grande-Bretagne au cours de laquelle des questions se posèrent au sujet de presque tous les aspects de la culture et de la société. Une gamme quasi infinie de réponses furent apportées de toutes parts. Ce sont ces questions et réponses qui se reflètent de manière si intéressante dans l'énorme production littéraire qui a saturé le marché dans les années 1790 et au début des années 1800, et qui a, seulement ces derniers temps, commencé à être appréciée dans toute son abondance.

La Révolution française et la poésie britannique

7 Pour l'étudiant à la recherche d'une vue d'ensemble rapide sur les interactions entre les poètes britanniques et la Révolution française, il existe plusieurs bons points de départ. Des introductions solides sont ainsi fournies par des chapitres uniques tels « Poetry in an Age of Revolution» de P.M.S. Dawson, par le court essai de Iain Robertson Scott " "Things As They Are": the Literary Response to the French Revolution », et par le chapitre de W.A. Speck: "Poems on the State of Affairs » dans son ouvrage Literature and Society in Eighteenth-Century England. Deux autres introductions, un peu plus complètes, destinées aux étudiants de niveau licence, se trouvent dans English Romantic Poetry: An Introduction to the Historical Context and the Literary Scene de Kelvin Everest et England and the French Revolution de Stephen Prickett qui est autant une anthologie d'extraits appropriés qu'un commentaire critique de bonne tenue. Prickett insiste sur le fait que les poètes lakistes modérèrent peu à peu leur soutien à la Révolution française, et accorde une plus grande importance à leur utilisation nuancée de la langue pour interpréter les événements de l'autre côté de la Manche.

8 À partir de ces introductions générales, l'étudiant pourrait souhaiter aborder l'étude de poètes romantiques particuliers et de leurs rapports spécifiques au contexte politique. Ceci serait certainement assez facile soit pour Blake, soit pour Wordsworth. Une analyse récente de leurs deux carrières illustre joliment les nouvelles directions que la critique historiciste prend à l'heure actuelle. Une récente étude qui donne à réfléchir est Dangerous Enthusiasm: William Blake and the Culture Radicalism in the 1790s de Jon Mee; elle est entièrement consacrée à la relation de Blake avec la crise de la Révolution. Une question que les critiques se sont toujours posés à propos de Blake est de savoir s'il devrait plutôt être considéré comme un activiste politique et social, ou comme un écrivain mystique dont les œuvres auraient aussi bien pu être écrites dans 
n'importe quel autre contexte. Mee insiste sur la première proposition, situant les écrits de Blake dans une tradition révolutionnaire. Ou plutôt dans plusieurs traditions révolutionnaires, car pour Mee, Blake est un «bricoleur* " qui puisait dans tout ce qui était disponible pour s'en inspirer. Ainsi, d'après Mee, il prit des éléments dans la culture populaire pour ses idées et sa rhétorique, de même qu'il en retira des idées dissidentes issues des Lumières, plus intellectuelles, qui influencèrent Coleridge et Godwin 7 . La discussion dans « l'Introduction » de Mee, d'un livre plus ancien sur Blake, celui de David Erdman Blake: Prophet Against Empire, montre très bien les changements qui ont eu lieu dans la critique littéraire historiciste au cours du dernier demi-siècle. Alors qu'Erdman s'efforçait de lier Blake à son contexte révolutionnaire, ainsi que son sous-titre l'indique, il montra l'influence directe de certains événements historiques sur les écrits. Mee voulait aller plus loin, par l'étude du langage, de la rhétorique et des formes poétiques de Blake, comme si en eux-mêmes ils répondaient à la crise des années $1790^{8}$.

9 La nature précise de l'extrêmisme de Blake a fait l'objet de nombreux débats. Le livre William Blake and the Impossible History of the 1790s de Saree Makdisi, comme celui de Mee, tente de distancier Blake du courant révolutionnaire dominant, du « combat pour la liberté et les "droits de l'homme" contre l'ordre héréditaire, politique et religieux, de l'ancien régime" ${ }^{9}$. Dans cet ouvrage à l'argumentation soignée, Blake est présenté comme dédaignant l'idée de liberté, comme si par liberté on entendait la notion bourgeoise de droits individuels et les libertés d'une économie capitaliste. Pour faire bonne mesure, Makdisi ajoute une tournure orientaliste à son analyse. The Chained Boy: Orc and Blake's Idea of Revolution de Christopher Z. Hobson fournit également une critique des suppositions établies depuis longtemps au sujet de l'idée qu'avait Blake de la révolution. Il est habituel, dans un compte rendu sur la carrière de Blake, d'indiquer qu'il en était venu à rejeter la révolution entre le début et le milieu des années 1790, estimant qu'elle devait inévitablement glisser vers un nouveau régime réactionnaire, encore pire que celui qui avait été remplacé. Une telle lecture conforte la thèse de M.H. Abrams selon laquelle la désillusion causée par la Révolution française obligea les poètes à intérioriser leurs impulsions révolutionnaires, ce qui donna lieu aux immenses débordements d'imagination qui constituent et définissent le romantisme. Pour Hobson cependant, la poésie de Blake du début des années 1790 révèle un changement dans sa compréhension des buts et des possibilités d'une révolution, mais pas un rejet total. Sous cet éclairage, ses travaux ultérieurs peuvent être lus comme politiquement aussi radicaux que ses premiers écrits idéalistes. Parmi de nombreuses autres études plus courtes sur l'interaction de Blake avec son contexte politique, on pourrait faire mention de «The "Ancient Voices" of Blake's The French Revolution» de Lisa Plummer Crafton et «Through Forest of Eternal Death: Blake and Universal History » de Barton Friedman (dans son ouvrage Fabricating History).

10 Les recherches concernant la relation de Wordsworth avec la Révolution sont tout aussi nombreuses. Dans les années 1980 parurent au moins quatre études importantes sur sa conception politique : Wordsworth and Coleridge: The Radical Years de Nicholas Roe, Wordsworth: The Sense of History d'Alan Liu, Wordsworth's Second Nature: A Study of the Poetry and Politics de J.K. Chandler, qui traita plus particulièrement de l'influence d'Edmund Burke sur Wordsworth, et, peut-être celle qui fait le plus autorité, Wordsworth's Great Period Poems de Marjorie Levinson. Levinson présenta également une théorie intéressante sur la relation entre l'histoire et la littérature dans son ouvrage Rethinking Historicism, mais ce fut sa lecture nouvelle de poèmes qui ostensiblement 
n'étaient pas politiques, tels l'ode "Intimations of Immortality " et dans un livre antérieur "Tintern Abbey " qui eut l'effet le plus durable. Pour elle, les événements politiques furent essentiels dans la forme que prit la poésie de Wordsworth, même lorsqu'ils étaient en apparence absents. Le choix des images pourrait révéler ce lien; d'après Levinson, la mention apparemment innocente d'un « arbre » solitaire pourrait faire allusion à l'arbre de la liberté en France, et celle du « champ unique » au Champ de Mars. Mais les omissions étaient plus importantes; elles représentaient le refoulement délibéré de l'optimisme politique et son remplacement par un nouveau système de croyances, le "mythe de l'âme » au centre de l'ode "Intimations ». Pour le Wordsworth désenchanté qui s'accrochait à de nouveaux idéaux dans la poésie, « il y a des victoires, écrit Levinson, bien plus grandes que celles jadis escomptées de la Révolution française. Plutôt que pleurer ces pertes prosaïques, le lecteur est incité à porter ses regards vers ces autres lauriers spirituels [...] les "pensées apaisantes qui jaillissent / de la souffrance humaine" " ${ }^{10}$.

11 Une telle interprétation aide à expliquer, voire à autoriser, l'abandon quasi désespéré par Wordsworth de son idéalisme de jeunesse. D'après David Bromwich, par exemple, l'absence d'un quelconque engagement politique clair dans un poème tel que Tintern Abbey est resté un point-clé, non parce que cette absence est l'indication d'une quelconque position politique cohérente, mais parce qu'elle montre l'évolution de "l'humanisme ", l'acceptation de l'intégrité individuelle, qui devaient caractériser les œuvres ultérieures de Wordsworth (Disowned by Memory: Wordsworth's Poetry of the 1790s; voir plus particulièrement le chapitre "The French Revolution and "Tintern Abbey" »). En d'autres termes, les événenements en France suscitèrent la recherche de sens nouveaux, et bien que les réponses proposées par Wordsworth sur le tard ne fussent pas nécessairement politiques en elles-mêmes, elles n'en étaient pas moins une réaction à la Révolution. Prise dans ses dimensions les plus extrêmes, ce genre d'insistance sur la politisation dé-politisée de la poésie de Wordsworth peut sembler quelque peu exagérée. «J'ai essayé de montrer comment le désengagement ostensible de la poésie de Wordsworth par rapport à l'action sociale et politique peut être analysé comme sa façon unique de s'engager dans l'extrêmisme politique ", écrit Yu Liu dans Poetics and Politics: The Revolution of Wordsworth; par son refus de prôner une quelconque position politique, Wordswords incitait ses lecteurs à se joindre à lui pour construire une nouvelle manière de comprendre le monde, plus éclairée ${ }^{11}$.

Certes, la réaction de Wordsworth vis-à-vis de la Révolution est extrêment compliquée. Trois essais de Kenneth R. Johnson, Jonathan Wordsworth et Anne Janowitz, bien qu'ils cohabitent sans se gêner dans le volume Revolution and English Romanticism, montrent combien de voies différentes peuvent être empruntées pour aborder le changement constaté chez Wordsworth à partir «d'une conscience révolutionnaire pour aboutir à une créativité imaginative $"{ }^{12}$. Nombre d'autres chercheurs continueront sans doute à explorer le sens de son retrait politique et de son reniement. Une étude récente, Romantics and Renegades de Charles Mahoney, est consacrée à cette "apostasie». Wordsworth semble avoir compris lui-même les complexités de sa position, remaniant avec habileté l'exposé de ses opinions politiques passées dans The Prelude, allant même jusqu'à falsifier le récit historique, ainsi que James A.W. Hefferman le note dans "History and Autobiography: The French Revolution in Wordsworth's Prelude" ${ }^{13}$. Pour certains commentateurs, les réactions de Wordsworth à la Révolution ne furent pas du tout motivées par ses convictions politiques, mais devraient être considérées comme les sublimations de traumatismes psychologiques profondément ancrés en lui ; 
c'est le cas de l'essai de Keith Hanley " "A Poet's History": Wordsworth and Revolutionary Discourse ».

On ne trouve pas tout à fait la même profusion d'études sur les effets de la Révolution française sur les œuvres d'autres poètes, bien que de bons travaux existent pour la plupart des figures de premier plan. Les tentatives pour suivre l'évolution du pessimisme de Coleridge après la Révolution n'ont pas été aussi nombreuses que pour Wordsworth. L'opinion de Nicholas Roe selon laquelle «la prise de conscience par Coleridge d'un effondrement total de l'espérance en France et en Grande-Bretagne ne trouve aucune alternative compensatoire qui pourrait ressembler aux grandes professions de foi de Wordsworth dans "Tintern Abbey" " est encore très plausible ${ }^{14}$. Pour Southey, la meilleure étude de ses écrits politiques reste l'ouvrage que Geoffrey Carnall leur a consacré : Robert Southey and his Age, mais une réévaluation est en cours. Une édition spéciale du journal sur l'internet, Romanticism on the Net (numéros 32-33), comportait un essai de Ian Haywood qui examinait la poésie révolutionnaire de Southey dans les années 1790, en particulier Joan of Arc (et la pièce Wat Tyler) où le poète imaginait le renversement d'un État britannique violent, un autre de Carol Bolton sur le poème narratif Thalaba the Destroyer (Thalaba le Destructeur) qui, d'après elle, présentait toujours une critique radicale de la société britannique derrière son programme impérialiste, et un troisième de Simon Bainbridge qui comparait les réactions de Southey et de Wordsworth aux événements politiques. "Robert Southey and the Meanings of Patriotism» de David Eastwood est un autre article important sur les convictions politiques de Southey.

14 L'influence de la Révolution française sur la génération suivante celle des grands poètes romantiques, a également été bien explorée. Dans une édition spéciale de Studies in Romanticism éditée par Susan Wolfson en 1986, «Keats and Politics: A Forum » était le signe d'une réévaluation de Keats en tant que radical, dont les écrits faisaient suite aux controverses des années 1790. John Keats and the Culture of Dissent de Nicholas Roe a fait plus encore pour rétablir ces liens. La réaffirmation d'un programme révolutionnaire après Waterloo, fondé sur les espoirs contrariés des années 1790 , fut à l'origine, d'après Jeffrey Cox dans Poetry and Politics in the Cockney School, du cercle qui se forma, pour une cause commune, autour de Leigh Hunt et inclut Keats, Shelley et de nombreux autres écrivains. La relation de Shelley avec la Révolution a été examinée par Timothy Clark dans Embodying Revolution: The Figure of the Poet in Shelley et par William Keach dans Arbitrary Power, Romanticism, Language, Politics. Dans Romance and Revolution: Shelley and the Politics of a Genre, David Duff enracine encore plus profondément la poésie de Shelley dans les controverses de la "crise de la Révolution", en étudiant les continuités linguistiques entre les tracts politiques des années 1790 et les poèmes narratifs de Shelley, et en explorant la manière dont Shelley et d'autres s'étaient approprié le poème narratif de chevalerie, visionnaire - en apparence peu susceptible d'être un vecteur de sentiments révolutionnaires - pour leurs opinions radicales.

En conformité avec les nouveaux principes historicistes, des poètes de moindre renom ont également fait l'objet de débat. Le lien entre Robert Burns et les événements en France a été brièvement évoqué dans Boswell, Burns and the French Revolution de Thomas Crawford. Les convictions politiques de John Clare, en particulier dans leur rapport avec l'extrêmisme dans les campagnes, ont été examinées dans English Literature in History 1780-1830 de Roger Sales et dans l'essai de James McKusick sur « William Cobbett, John Clare and the Agrarian Politics of the English Revolution ». Ce dernier essai se 
trouve dans Radicalism in British Literary Culture, ouvrage qui établit les continuités entre la tradition radicale de l'Angleterre au XVIIe siècle et les controverses suscitées par la Révolution française. Dans ce même volume figure l'essai de Jon Mee «The Strange Career of Richard "Citizen" Lee: Poetry, Popular Radicalism and Enthusiasm in the 1790 s", qui est important en tant que présentation d'un poète "Jacobin " presque oublié. Lee était étroitement lié à la "London Corresponding Society ", une association radicale; il possédait une boutique appelée "The Tree of Liberty ", et il était, d'après Mee, «le fournisseur de textes les plus ouvertement séditieux à Londres" ${ }^{15}$. Il fut bientôt arrêté; sa poésie est de qualité contestable, mais l'analyse de Mee qui place Lee dans une longue tradition révolutionnaire et religieuse, pas très loin de Blake, aide à éclairer d'un jour nouveau le monde trouble de la littérature radicale clandestine qui fut pour la première fois portée à notre attention par Radical Underworld: Prophets, Revolutionaries and Pornographers in London de Iain McCalman. L'essai de Charles Hobday sur deux autres "poètes sans-culottes", John Freeth et Joseph Mather, accomplit la même tâche.

Un autre poète qui débuta comme écrivain révolutionnaire, mais dériva vers la passivité politique fut Amelia Opie. L'étude de Shelley King, "Politics, Poetics and Propriety: Reviewing Amelia Opie », porte sur la manière dont ses poèmes avaient été accueillis par ses contemporains et soutient que Opie avait été manipulée par les critiques pour revenir à l'orthodoxie politique, bien que l'on puisse toujours déceler l'extrêmisme sous la surface. L'essai de Ann Frank Wake sur Opie dans Rebellious Hearts: British Women Writers and the French Revolution pose également la question de savoir pourquoi « elle cacha ou travestit ses vues politiques dissidentes dans sa poésie » (alors que ses lettres révèlent " une participation plus enthousiaste à la politique Jacobine ») ${ }^{16}$. La réponse de Wake est que les femmes écrivains risquaient d'être diabolisées pour avoir exprimé des opinions politiques radicales dans la Grande-Bretagne des années 1790, mais, comme Kari E. Lokke qui examine les sonnets de Charlotte Smith dans le même ouvrage, elle conclut que les femmes poètes avaient la possibilité d'utiliser la représentation poétique du paysage pour transcender les codes restrictifs contre les femmes et communiquer leurs opinions politiques.

Toutes les analyses n'ont pas porté sur des voix individuelles. Richard Cronin, par exemple, propose un bon exposé sur la réaction poétique en Grande-Bretagne à la Révolution dans son ensemble dans The Politics of Romantic Poetry: In Search of a Pure Commonwealth. Son «Introduction » fournit un résumé utile des travaux récents dans ce domaine, et les chapitres suivants montrent de façon convaincante comment les problèmes politiques des années 1790 restèrent d'actualité pour de nombreux auteurs jusque dans les années 1810 et 1820 . Un autre ouvrage pour faire la synthèse des réponses exprimées par de nombreuses voix est celui de Simon Bainbridge, qui, pour British Poetry and the Revolutionary and Napoleonic Wars, a retrouvé la poésie de guerre produite tant par les poètes qui font autorité que par d'autres, publiée seule ou dans des journaux et magazines. Bainbridge présente un double argument : que les guerres avec la France eurent un effet considérable sur la forme de la production poétique en Grande-Bretagne, et que les poèmes de guerre écrits par des personnalités telles que Coleridge et Byron, Charlotte Smith et Felicia Hemans eurent également un effet immense sur la formation des attitudes du public vis-à-vis des guerres.

La Révolution française et le théâtre 
18 Les recherches sur les effets de la Révolution française sur le théâtre en GrandeBretagne ont été, dans le passé, bien moins nombreuses que pour la poésie et la prose. Ceci a récemment changé. L'étude de Jeffrey Cox, "The French Revolution in the English Theater », constitue maintenant un bon point de départ. Dans l'analyse qu'en fait Cox, l'engagement du théâtre britannique vis-à-vis de la Révolution française est allé «de la représentation directe de faits réels au déplacement d'actes révolutionnaires vers des parallèles néoclassiques et gothiques et finalement à la recréation de la Révolution en termes mythiques »; en d'autres termes, sous la pression de la censure, la Révolution française est devenue, de manière de plus en plus indirecte, un élément dans la production théâtrale ${ }^{17}$. Ainsi, bien que (selon Kenneth Johnson et Joseph Nicholes, dans un autre essai d'introduction utile) seule une pièce "par un écrivain romantique anglais de premier plan se passe dans la France révolutionnaire »The Fall of Robespierre (1794) de Coleridge et Southey - nombreuses sont les œuvres qui traitent de questions révolutionnaires, mais dans un cadre différent. La plus marquante d'entre elles est The Borderers (Les Frontaliers) de Wordsworth (écrite en 1796-97): «la forme adaptée pour la scène des réflexions que Wordsworth ne cessait de se faire sur la révolution en France " ${ }^{18}$. Pour Cox, la réécriture gothique de la Révolution est illustrée par la pièce The Castle Specter (1797) de Matthew Lewis, qui plut énormément au public, dans laquelle les protagonistes, jeunes et optimistes, tentent de s'échapper d'un château qui se délabre et qui « semble symboliser un passé aristocratique en ruines » ${ }^{19}$. Cox suggère que les itérations néo-classiques de la Révolution atteignirent leur apogée avec Marino Faliero, The Two Foscari ou Sardanapalus de Byron (toutes de 1821).

19 La première étude de grande ampleur sur ce sujet, The French Revolution and the London Stage, 1789-1805, de George Taylor, suit ce même raisonnement dans l'ensemble. Taylor montre que bien que les plus petits théâtres aient pu éviter les restrictions imposées par le grand chambellan au moment où la Révolution française éclata, et avaient donc eu la possibilité de présenter des adaptations pour la scène des événements de Paris pleines d'enthousiasme, la censure fut rapidement imposée pour tous les théâtres londoniens. Ceci obligea ceux qui s'étaient engagés en faveur de la Révolution française à être plus discrets. La censure signifiait que les écrivains radicaux «devaient abandonner le théâtre comme moyen rationnel d'instruction ", et le commentaire politique se trouva relégué dans des formes théâtrales apparemment peu adéquates: divertissements musicaux, pantomimes, et surtout, nouvelle manifestation du gothique, le mélodrame ${ }^{20}$. Au cours des années 1790 Taylor indique que les grandes inquiétudes politiques et les espérances étaient représentées par la métaphore; les images d'emprisonnement, la cécité ou la spoliation étaient habituellement utilisées pour faire référence, sous forme voilée mais reconnaissable, aux événements en cours.

20 Les pièces de théâtre du début de la Révolution française, qui étaient vraiment en prise directe sur les événements de l'époque, n'ont pas encore été étudiées à fond. Des œuvres telles que Democratic Rage; or Louis the Unfortunate (1793) de William Preston, ou de John Eyre Maid of Normandy; or, the Death of the Queen of France (1794) soit reprenaient dans leur intégralité des événements qui avaient eu lieu en France, soit les incluaient pour figurer dans une intrigue inventée, alors que d'autres, telles St. Kilda in Edinburgh; or, News from Camperdown (1798) de Robert Heron s'attaquaient au vice et à la folie de ceux qui, en Grande-Bretagne, étaient partisans de la Révolution française. Il existe une telle quantité de pièces de ce genre que Taylor, comme Cox dans son article «Ideology and Genre in the British Antirevolutionary Drama of the 1790s ", ne peut donner qu'un 
aperçu fort alléchant de leur portée et de leur variété. Même des pièces, qui connurent le succès, écrites par des auteurs célèbres - par exemple Pizarro (1799) de Richard Brinsley Sheridan, œuvre très appréciée adaptée de Kotzebue qui très tôt attaque Napoléon et fait mouche - sont encore bien négligées ${ }^{21}$. Le livre de Paula Backscheider Spectacular Politics est celui qui va le plus loin dans ses investigations sur la signification culturelle et politique des pièces gothiques, également très délaissées - alors que c'était un « mode littéraire très prisé » dans les années 1780 et une " passion » dans les années $1790{ }^{22}$. Dans l'analyse de Backscheider, ces pièces auraient pu être d'inspiration radicale, dépeignant maintes et maintes fois des aristocrates infâmes qui ne respectaient pas la vie, la liberté, la propriété et l'honneur des femmes, et des foules puissantes capables de les renverser - tout ceci évidemment en rapport avec la situation politique des années 1790. Mais, ajoute-t-elle rapidement, « au fur et à mesure des années, les pièces se caractérisent par des stratégies d'endiguement de plus en plus vigoureuses ». Elles décrivent la loi de la rue comme étant tout aussi préjudiciable que le despotisme et représentent les protagonistes vertueux et dans leur bon droit comme capables de vaincre les abus et de restaurer l'ordre par des moyens non conflictuels. Puis finalement ces pièces " propageaient une vision réconfortante d'une communauté, de la nature humaine, et d'un monde providentiel organisé avec bienveillance». Plus important encore, elles constituaient une soupape de sécurité pour un public inquiet et agité:

«Parce qu'elles exprimaient sur scène des appréhensions et des espoirs refoulés sans ambages et de manière symbolique, les pièces de théatre relâchaient les tensions et rendaient les contradictions pour un temps anodines. L'antagonisme social se transformait en occasions de montrer l'image idéalisée que les Britanniques avaient d'eux-mêmes et de confirmer qu'aucune révolution n'était nécessaire dans leur pays. [...] Il serait possible de contenir les aristocrates et on s'occuperait des pauvres. [...] Le conflit devint spectacle, produit maîtrisable et consommable». ${ }^{23}$

21 Cox de même que Backscheider souligne la nature anti-révolutionnaire et conservatrice de nombreuses pièces de théâtre appréciées à Londres dans les années 1790. D'autres commentateurs ont trouvé que certains textes relevaient de l'inspiration radicale. L'article de Ian Haywood, " "The Renovating Fury": Southey, Republicanism and Sensationalism", concerne Wat Tyler, pièce pro-révolutionnaire. A Materialist Critique of English Romantic Drama de Daniel P. Watkins étudie DeMontfort (1798) de Joanna Baillie et affirme que l'intrigue de cette pièce trouve ses origines dans l'agitation politique post-révolutionnaire, et tourne autour du transfert de pouvoir de DeMontfort, aristocrate despotique, au bourgeois Rezenbelt ${ }^{24}$. L'article de Katherine S. Green, « Mr Harmony and the events of January 1793 ", a pour objet la pièce d'Elizabeth Inchbald, Every One Has His Fault, le succès de la saison 1793, en dépit du fait que sa première ait eu lieu juste après l'exécution des roi et reine de France (la représentation fut reportée à cause du régicide, et lorsqu'elle eut lieu, presque tout le public était en deuil). D'après Green, le fait que cette pièce ait été donnée pendant trente-deux soirées fut étonnant, car elle " apportait à la fois son soutien à une idéologie réformiste et se lançait dans la critique du texte Enquiry Concerning Political Justice (Enquête sur la justice politique) de William Godwin ». Inchbald dut user de stratégies subtiles pour faire illusion afin de s'assurer la faveur du public ${ }^{25}$. Dans son essai, «"The Conflict”: Hannah Brand and theatre politics in the 1790s ", David Chandler recherche aussi les manières dont un auteur dramatique pouvait imposer des sentiments révolutionnaires dans une intrigue en apparence orthodoxe. Le principal censeur de Brand à Norwich n'était pas 
le grand chambellan, mais John Brunton, directeur de théatre d'un conservatisme rigide, dont le soutien était nécessaire si tant est que ses pièces devaient être jouées. En fait, de nombreux écrits de femmes pour le théâtre sont à l'heure actuelle considérés comme révolutionnaires de différentes façons, peut-être parce qu'ils contestent l'orthodoxie en matière de rôles selon le sexe, ou l'image que la nation avait d'ellemême; mais ils ne participent pas toujours directement au débat sur la Révolution française (voir par exemple Women, Nationalism and the Romantic Stage de Betsy Bolton). Une vue d'ensemble des études de spécialistes concernant les pièces de théâtre écrites par des femmes se trouve dans l'essai critique : « Revising Romanticism by Inscripting Women Playwrights », de Marjean D. Purinton.

Purinton a également écrit au sujet de l'aspect politique du théâtre romantique, en attirant une attention particulière sur les représentations du despotisme dans Romantic Ideology Unmasked: the mentally constructed tyrannies in dramas of William Wordsworth, Lord Byron, Percy Shelley, and Joanna Baillie. Les discussions sur les pièces des auteurs romantiques de premier plan, de même que sur leur poésie, ont porté sur la manière dont les débats sur la Révolution française dans les années 1790 ont aussi fait irruption dans ce genre nouveau. L'essai de Purinton, "The English pamphlet war of the 1790s and Coleridge's Osorio ", correspond à ce schéma, tout comme celui de Victoria Myers : "Justice and Indeterminacy: Wordsworth's The Borderers and the Trials of the 1790s ". De la même manière, Michael Simpson affirme que dans les pièces de Byron et Shelley, les débats des années 1790 sur la révolution étaient «matérialisés" dans la forme imprimée - assurant ainsi, écrit Simpson, qu'ils continueraient à être seulement l'objet de réflexions et de discussions plutôt qu'être vraiment représentés sur scène. Ce scepticisme, d'après Suzanne Ferriss, est on ne peut plus clairement exprimé dans la pièce de Shelley The Cenci (1819), ce qui « jette un doute sur la possibilité que les idées révolutionnaires trahies en France par la Terreur et l'accès au pouvoir de Napoléon puissent être récupérées d'un point de vue soit politique, soit poétique ${ }^{26}$.

Jeffrey Cox a émis l'idée qu'au « cours des journées impressionnantes et terribles de la Révolution française, l'histoire elle-même semblait devenir théâtrale », et de nombreux critiques, dont la pensée suit cette direction, ont exploré la façon dont la politique britannique en était venue à être représentée comme une pièce, que ce soit dans les théâtres et au dehors ${ }^{27}$. Les discours d'Edmund Burke à la Chambre des communes et les procès pour trahison de 1794, pour ne prendre que deux exemples, relevaient, les uns comme les autres, de l'art dramatique (ainsi que Gillian Russell et Judith Pascoe en font l'étude respectivement dans l'essai «Burke's Dagger » et le chapitre "Courtroom Theatre » de Romantic Theatricality [ $\left.{ }^{28}\right]$ ). Et, bien entendu, l'expérience d'assister à une représentation dépassait de beaucoup le seul texte des pièces qui étaient données en ces lieux. Le public jouait un rôle plein à chaque représentation, donnant le ton à la pièce ; les spectateurs communiquaient avec les acteurs, et entre eux, de manière telle que souvent la pièce elle-même était reléguée à la périphérie de cette expérience. Toute l'activité et tous les textes autour des pièces contribuaient au spectacle : les décors et le prix des places, les publicités et les critiques, la claque et la foule restée au dehors, même le bâtiment lui-même. La représentation de la politique au théâtre dans ce sens plus large est l'objet de plusieurs ouvrages importants. L'un des plus aboutis est Theatres of War de Gillian Russell, qui observe le spectacle de la Révolution française et des guerres napoléoniennes sur la scène de théâtres publics et privés, ainsi que la présence de militaires dans le public et ses conséquences, et les représentations 
théâtrales par des militaires dans des casernes ou à bord de navires (on y trouve également une enquête substantielle sur le caractère théâtral des funérailles de Nelson). Marc Baer dans Theatre and Disorder in Late Georgian London étudie aussi comment les tensions politiques trouvaient un exutoire dans l'enceinte du théâtre et ses alentours.

La Révolution française et le roman

Depuis les travaux de Marilyn Butler (Jane Austen and the War of Ideas) et de Gary Kelly (The English Jacobin Novel) qui, au milieu des années 1970, ont ouvert de nouvelles voies, une quantité considérable de recherches ont tenté de découvrir les rapports qui ont existé entre le roman britannique de la fin du XVIIIe siècle et du début du XIXe et la Révolution française, la crise qu'elle a causée, mais aussi la façon dont le roman les a reflétés et comment il y a contribué. Butler et Kelly montrèrent tous deux comment les romanciers en Grande-Bretagne dans les années 1790 jouèrent un rôle central dans la "guerre des idées » déclarée presque tout de suite après le début de la Révolution française. Il ne s'agissait pas de romans au sujet de la Révolution, mais ils étaient certainement de la Révolution. Ils se lançaient sans réserve dans les débats sur les droits, les libertés et même la possibilité de renverser un régime corrompu que les événements de France avaient fait naître ${ }^{29}$. Par la suite, les spécialistes ont fait surtout porter leurs études sur les romanciers radicaux - les «Jacobins » ainsi que Kelly les avait appelés. Des travaux ont observé leurs méthodes, leurs convictions et ce qui est arrivé lorsque leur utopisme initial s'est trouvé modéré ou étouffé. Kelly s'est intéressé en particulier à quatre romanciers radicaux : Robert Bage, Thomas Holcroft, William Godwin et Elizabeth Inchbald. D'autres ont ajouté de nouveaux noms. L'essai de Loraine Fletcher, « Four Jacobin women novelists », et The Autobiography of Desire: English Jacobin Women Novelists of the 1790s de Anjana Sharma incluent Mary Hays et Charlotte Smith, et The English Jacobin Novel on Rights, Property and the Law de Nancy E. Johnson ajoute Maria Edgeworth. Chris Jones les avaient déjà toutes citées dans son important ouvrage Radical Sensibility. Pour sa part, Pamela Clemit analysa la stylistique des romanciers « jacobins » et leur influence durable dans The Godwinian Novel, et, dans la même veine, "Godwin's Literary Theory» de David McCracken est utile. Tous ces commentateurs ont suivi les conclusions de Kelly pour penser que les romanciers jacobins contribuaient à former un genre cohérent. Ce genre est devenu si bien établi dans les études littéraires que, à l'heure actuelle, les spécialistes considèrent ces auteurs comme formant un groupe dans des études non liées à la crise de la Révolution française. Liz Bellamy, dans son ouvrage Commerce, Morality and the Eighteenth-Century Novel, inclut ainsi un chapitre "The Jacobin Novel» dans son analyse de la représentation de l'orientation commerciale de la société dans la fiction.

25 Il fallut davantage de temps pour que la fiction anti-jacobine devienne l'objet d'une analyse approfondie. Ce n'est qu'en 2001 que parut la première étude entièrement consacrée aux romans conservateurs qui participèrent à la guerre des idées. Dans The Anti-Jacobin Novel, M.O. Grenby souligne que les romans anti-jacobins étaient de loin plus nombreux que leurs équivalents jacobins et qu'ils commencèrent à paraitre un peu plus tôt (le premier roman britannique qui s'engage totalement vis-à-vis de la Révolution fut probablement Lindor and Adelaïde, a Moral Tale: In which are exhibited the Effects of the Late French Revolution on the Peasantry in France, de Edward Sayer, publié dès 1791). Mais il apporte peu pour contrer l'hypothèse selon laquelle les écrivains radicaux étaient plus profonds, plus novateurs, et donc de plus grande portée en termes de production littéraire sinon de documents historiques. Lisa Wood considère également 
les auteurs anti-jacobins comme constituant un genre cohérent dans Modes of Discipline: Women, Conservatism and the Novel after the French Revolution. Grenby et Wood mettent tous deux l'accent sur ce que Wood appelle «l'interarticulation des idéologies ", c'est-àdire l'établissement de liens entre les différentes campagnes conservatrices : contre la Révolution, le féminisme, les parvenus, et ainsi de suite. Toutes ces campagnes furent réunies par les romanciers anti-jacobins pour n'en faire qu'une et devenir ainsi un élément dans une seule conspiration jacobine qui pouvait alors être méprisée et faire l'objet de nombreuses satires. Grenby et Wood montrent, par exemple, comment les intrigues de séduction conventionnelle dans le roman pouvaient être mises à contribution pour décrire une conduite politique correcte. Ainsi, selon Wood, la séduction d'une Britannique par un personnage de nouveau philosophe jacobin, « représente-t-elle, au niveau de la vie privée, la pénétration de la philosophie française dans l'organisation sociale britannique. [...] La femme patriote résiste à cette pénétration, par son acceptation des normes d'une féminité pleine de réserve dans un cadre familial et le respect des doctrines de l'Église nationale établie ${ }^{30}$.

Nicola J. Watson, dans Revolution and the Form of the British Novel, étudie également les associations entre la forme du roman et la politique. Pour Watson, des romanciers des deux côtés dans le débat sur la Révolution se rendirent compte qu'il existait un lien évident entre quelques thèmes majeurs du roman et les discussions politiques des années 1790. L'évocation d'une héroïne de roman sentimental qui se choisit un amant au mépris de la décision de ses parents, par exemple, correspondait peut-être à la désobéissance vis-à-vis de l'autorité politique, de la même façon que la tentative de séduire une héroïne pouvait représenter les tentatives d'insurgés pour inciter des Britanniques sans méfiance à la sédition. Watson indique que les romanciers conservateurs aussi bien que les radicaux essayèrent d'exploiter ces relations métaphoriques, rendant ainsi les romans des années 1790 beaucoup plus politiques qu'il y pouvait paraître à première vue. Watson soutient que la forme même du roman était une déclaration politique en soi. Les romans épistolaires, par exemple, étaient fondamentalement politiques parce que la lettre était un objet tout à fait suspect dans les années 1790; elle représentait la communication secrète entre individus et était liée aux «sociétés de correspondances» radicales (créées en Grande-Bretagne pour communiquer directement avec les révolutionnaires en France), à l'espionnage, aux preuves utilisées dans les procès pour trahison ${ }^{31}$. Dans son essai dans The Cambridge Companion to British Romanticism, Gary Kelly signale aussi le lien entre la forme et le style de ces romans et leur orientation politique. Les anti-jacobins étaient hostiles aux récits subjectifs à la première personne, d'après lui, car c'était la technique prisée des radicaux. Il ajoute que les anti-jacobins faisaient beaucoup appel à différentes techniques satiriques figées; ils mettaient en opposition la théorie idéalisée et la "vraie vie » telle qu'elle se déroulait dans les intrigues des romans, par exemple. Les deux articles d'April London, « Novel and History in Anti-Jacobin Satire » et « Clock Time and Utopia's Time in Novels of the 1790s ", fournissent des prolongements intéressants sur ce point. Selon James Watts, dans Contesting the Gothic: Fiction, Genre and Cultural Conflict, "presque tous les romans qui en fait se disent "gothiques" sont sans ambiguité conservateurs ». Le conservatisme était ancré dans leurs structures mêmes, et non pas délibérément importé par des auteurs tenants d'une idéologie. En général, il y avait un château, qui représentait "une société stratifiée et pourtant harmonieuse", et l'intrigue dramatique portait sur « la défaite de scélérats équivoquement efféminés ou 
étrangers» et «le processus inspiré par la providence par lequel les hiérarchies légitimes sont rétablies ${ }^{32}$.

Tout comme le roman jacobin, le roman anti-jacobin a récemment commencé à être traité comme un genre littéraire cohérent. April London, par exemple, inclut un chapitre "History, Romance and the Anti-Jacobins' "Common Sense" » dans son livre Women and Property in the Eighteenth-Century English Novel, et une collection de dix romans anti-jacobins est en cours de préparation chez Pickering and Chatto, sous la direction générale de Wil Verhoeven. Rien de comparable n'est à paraître pour les romans jacobins, en grande partie parce que les œuvres des meilleurs romanciers jacobins, en opposition à leurs adversaires conservateurs, ont souvent été considérées comme dignes d'être publiées séparément. Ainsi Pickering and Chatto ont ou auront publié des collections de romans de William Godwin, Mary Wollstonecraft et Thomas Holcroft ainsi que de Maria Edgeworth et Charlotte Smith, plus ambigües d'un point de vue politique. Les romans jacobins pris individuellement ont été publiés un par un par des éditeurs plus traditionnels. La série des Penguin Classics, par exemple, comprend Caleb Williams de Godwin, Mary et Maria de Wollstonecraft, alors que parmi les titres publiés dans les Oxford World's Classics on peut trouver Caleb Williams, Memoirs of Emma Courtney de Mary Hays, Adeline Mowbray d'Amelia Opie, et The Wanderer; or, Female Difficulties de Frances Burney. L'intérêt croissant des spécialistes pour ces romans britanniques qui s'engagèrent vis-à-vis de la Révolution française se trouve peut-être mieux reflété - et servi - par la liste en expansion rapide de la Canadian Broadview Press. Vers la fin des années 1990, cette société a commencé par publier, généralement pour la première fois depuis leur sortie initiale dans les années 1790, plusieurs textes révolutionnaires, tels que Desmond de Charlotte Smith, Hermsprong or, Man as He Is Not de Robert Bage, Letters written in France d'Helen Maria Williams, The Victim of Prejudice et Emma Courtney de Hays, Walsingham; or, the Pupil of Nature de Mary Robinson et Secresy d'Eliza Fenwick. Plus récemment, et peut-être même plus courageusement, Broadview a sorti un certain nombre de romans anti-jacobins complètement laissés de côté, parmi lesquels se trouvent Letters of a Hindoo Rajah et Memoirs of Modern Philosopher d'Elizabeth Hamilton, The Infernal Quixote de Charles Lucas et The Vagabond de George Walker.

28 Ainsi que George Walker l'indique clairement au début de The Vagabond, les romans anti-jacobins étaient écrits en opposition ouverte aux romans jacobins, comme " tentative pour parer les coups de l'ennemi avec ses propres armes " ${ }^{33}$. Les lecteurs et critiques contemporains reconnaissaient l'existence de deux écoles politiques dont les opinions s'affrontaient dans les pages du roman. Mais ceci n'a pas empêché les spécialistes de débattre sur le fait que la dichotomie jacobin/anti-jacobin a été exagérée. Claudia Johnson indique que cette opposition était plus rhétorique que réelle, et qu'il y avait peu d'accord à l'intérieur des deux principaux camps:

"La plupart des romans écrits au cours de la "guerre des idées" sont plus complexes et moins doctrinaires que les commentateurs modernes les ont représentés. Il ne suffit pas de qualifier des écrivains de "conservateurs" ou "révolutionnaires" selon qu'ils étaient "pour" ou "contre" la Révolution française. Vers le milieu des années 1790, quand la France et l'Angleterre étaient en guerre et que la Révolution et la Terreur étaient des faits accomplis, il y avait peu de "jacobins" Anglais, et parmi ceux qui se disaient "anti-jacobins" il existe beaucoup plus de désaccords qu'il ne semble à première vue ". ${ }^{34}$

Que l'on soit d'accord sur l'utilité ou non des termes «conservateur» et « révolutionnaire », ou « jacobin » et « anti-jacobin », il est certain que les nuances de 
sens, voire les contradictions dans ces positions de base peuvent donner lieu à un examen fructueux. Ainsi que l'a remarqué April London dans une critique de romans de Charlotte Smith et d'Elizabeth Hamilton - deux écrivains déclarés comme à la fois révolutionnaires et conservateurs -, "des éléments apparemment incompatibles d'opinions orthodoxes et iconoclastes peuvent coexister sans se gêner, et parfois de manière créative, dans un même ouvrage $"{ }^{35}$. Cette idée apparemment paradoxale d'un conservatisme révolutionnaire (ou d'ailleurs d'un radicalisme conservateur) n'a pas été complètement étudiée dans les analyses de ces romans écrits par des femmes dont on pourrait dire qu'ils prônent une réforme culturelle, et plus particulièrement un défi aux vues patriarcales et anti-féministes, mais qu'ils soutiennent l'orthodoxie politique de manière explicite.

30 Les figures-clés qui apparaissent dans ce débat sont Hannah More, Jane West et Elizabeth Hamilton. Ces écrivains étaient farouchement hostiles à la Révolution française et au jacobinisme britannique qu'au moins More et West considéraient sans aucun doute comme impardonnablement irréligieux. Pourtant, on a donné des arguments solides selon lesquels, derrière ce conservatisme explicite se trouvait une détermination de cesser d'accorder aux femmes des rôles restrictifs, que Mary Poovey a décrite dans son ouvrage important: The Proper Lady and the Woman Writer. Ainsi dans Women Writing and Revolution, Gary Kelly place-t-il Hamilton - alors même qu'elle était l'auteur de deux romans politiquement conservateurs - aux côtés de Mary Wollstonecraft, Helen Maria Williams et Mary Hays, bien plus ouvertement féministes, toutes étant des détractrices du code patriarcal. De même, le livre d'Eleanor Ty, Empowering the Feminine, présente l'ostensiblement conservatrice Jane West comme une critique du patriarcat dans ses romans. Ses méthodes, ainsi que Ty les présente, étaient subtiles, et nombreuses sont celles qui ont échappé à certains contemporains, mais elles ont beaucoup à voir avec les stratégies utilisées par des écrivains politiquement plus ambigus comme Mary Robinson et Amelia Opie. Et, Ty le montre, West peut même être comparée à des auteurs ouvertement révolutionnaires comme Wollstonecraft, Hays, Williams, Inchbald et Smith qui, toutes, figuraient dans Unsex'd Revolutionaries, l'étude précédente de Ty. Dans cet ouvrage, Ty avait tenté d'expliquer pourquoi les femmes écrivains pouvaient se positionner comme conservatrices déclarées alors que, simultanément, elles mettaient en avant un programme radicalement féministe. Elles utilisaient, d'après Ty, ce que Mikhail Bakhtin avait appelé «hétéroglossie » ou "discours à deux voix». Ceci signifiait que l'auteur acceptait une position conservatrice, orthodoxe, mais en même temps proposait une approbation codée des idées qu'il dénonçait en surface, ou au moins une nouvelle réflexion ${ }^{36}$.

Tous les critiques n'ont pas approuvé ce genre d'analyse. Dans Their Fathers' Daughters, Elizabeth Kowaleski-Wallace a abordé les mêmes problèmes, mais son argument a été qu'Hannah More et Maria Edgeworth ont moins bravé le système patriarcal qu'elles n'en ont été complices. D'autres ont pris ce que l'on pourrait penser être une voie médiane, et ont suggéré qu'un personnage tel que More pouvait défier les éléments répressifs de l'idéologie des sphères séparées qui emprisonnait les femmes dans certains rôles restreints, mais sans obligatoirement attaquer le paradigme dans son intégralité. L'essai remarquable de Mitzi Myers, «Reform or Ruin ", tenta de montrer les similitudes entre les campagnes de deux voix que l'on supposait antithétiques: Wollstonecraft et More. Pour Myers, « des idéologies croisées (mais qui ne s'excluent pas l'une l'autre) concernant la vie familiale, telles que le modèle de féminité 
évangélique de More et la condition féminine rationnelle de Wollstonecraft, sont parallèles, voire symbiotiques; ce sont des réactions de femmes à la révolte politique, des tentatives pour tirer profit du malaise national afin de re-créer une vie familiale d'après de nouvelles images schématiques de l'ordre social $"{ }^{37}$. On pourrait argumenter que ces campagnes auraient pu être menées sans aucun rapport avec les événements de France, mais Myers prend soin de lier son analyse à la Révolution - dans les écrits de More et de Wollstonecraft se trouvent « des réactions féminines à la révolte politique ». Dans le livre d'Anne Mellor, Mothers of the Nation, More est encore une fois la figure-clé. D'après Mellor, elle mit au point une stratégie pour l'émancipation des femmes, précisément en les exhortant à affirmer leur domination dans la sphère privée, recommandant par ailleurs ces valeurs auparavant dépréciées pour qu'elles occupent le centre même du discours politique et public. More ne préconisait pas que les femmes transgressent les codes traditionnels, mais affirmait que dans la crise qui suivit la Révolution française, c'était précisément ces valeurs et vertus féminines traditionnelles qui devaient être placées au cœur de la nation. Mellor indique qu'elle rencontra un vif succès, et l'image que la nation avait d'elle-même au début de la période victorienne était celle "d'une femme rationnelle, juste mais clémente, vertueuse, bienveillante, et pacifique », la « Nouvelle Femme » que More avait contribué à inventer ${ }^{38}$. Deux autres analyses intéressantes sur la politique de la Révolution en matière de relations sociales entre hommes et femmes et sa description dans les romans sont celles de Julie Shaffer, «Ruined Women and Illegitimate Daughters: Revolution and Female Sexuality " (comprendre la sexualité féminine illicite, tolérée dans certains romans, comme représentant des actes politiques de libération féminine ou de classe) et celle de Lucinda Cole: "(Anti)Feminist Sympathies: The Politics of Relationship in Smith, Wollstonecraft, and More ».

Toutes les études de romans examinées jusque là ont eu pour objet un groupe d'auteurs pris ensemble, mais travailler sur des auteurs traités individuellement donne aussi des indications importantes, souvent moins théoriques, sur la manière dont la fiction répondait à la Révolution française et à ses conséquences. Il existe beaucoup trop d'études de ce genre pour que l'on puisse les prendre toutes en compte ici, mais une sélection représentative peut inclure Tilottama Rajan à propos de Mary Hays, Gary Kelly sur « Enlightenment and Revolution: The Philosophical Novels of Dr. John Moore» , Miriam L. Wallace concernant Mary Hays, Joseph Roseblum pour Thomas Hofland, Maria Jerinic pour Frances Burney, l'article de James Whitlark sur M.G. Lewis, « Heresy Hunting: The Monk and the French Revolution » et, de M.O. Grenby : «Orientalism and Propaganda " à propos de certains écrits jacobins et anti-jacobins plus courts, publiés sous forme de contes orientaux. Deux auteurs se détachent par rapport aux autres et ont de ce fait attiré la plus grande attention: Jane Austen, à cause de l'intérêt que tout le monde porte à tous les aspects de son œuvre, et Charlotte Smith, à cause de sa description très franche de la Révolution française dans certains de ses romans. Pour Austen, le texte-clé reste Jane Austen and the War of Ideas de Marilyn Butler, mais il a été rapidement rejoint par l'ouvrage de Warren Roberts, Jane Austen and the French Revolution, puis par une avalanche de livres et d'articles à ce propos à la fin des années 1980: de Mary Evans, Jane Austen and the State, de Claudia Johnson, Jane Austen: Women, Politics and the Novel, et de Gary Kelly, «Jane Austen and the English Novel of the 1790 » . Les auteurs de ces études prennent plaisir à saper l'affirmation de Winston Churchill selon laquelle tous les personnages d'Austen menaient une "vie paisible ", sans « aucun souci à propos de la Révolution française ou des guerres napoléoniennes ", 
affirmation à nouveau brièvement étudiée dans l'article de John Dussinger : "Jane Austen's Political Silence ${ }^{39}$.

Les romans de Charlotte Smith peuvent paraitre refléter parfaitement les attitudes changeantes de la société britannique dans son ensemble à l'égard de la Révolution française. Dans son livre, Desmond, de 1792, l'enthousiasme pour la Révolution est très vif. Old Manor House de 1793 semble en retrait; il se passe environ dix ans plus tôt et ne fait pas mention de la Révolution. Puis The Banished Man de l'année suivante revient à la Révolution française, désavouant semble-t-il le soutien passé et dépeignant la France post-révolutionnaire comme une société tombée dans l'anarchie et la barbarie. Les deux romans ultérieurs de Smith, Marchmont (1796) et The Young Philosopher (1798), traitent également de problèmes politiques mis en évidence par la Révolution française; tous deux semblent attaquer l'orthodoxie anti-jacobine de rigueur en Grande-Bretagne vers la fin des années 1790. Naturellement, les problèmes concernant les changements d'opinion politique de Smith sont traités dans deux biographies récentes par Loraine Fletcher et Carroll Lee Fry. Sa représentation de la Révolution et la question de savoir si ces descriptions doivent être prises au pied de la lettre seront également abordées dans les éditions prochaines de ses romans chez Pickering and Chatto. Peut-être le meilleur résumé disponible, à l'heure actuelle, est l'essai de Judith Davis Miller : «The Politics of Truth and Deception: Charlotte Smith and the French Revolution ».

34 L'œuvre de Smith nous rappelle que quelques romans représentaient sans détours la Révolution en France au lieu de simplement traiter des controverses idéologiques qui en avaient résulté. Dans son essai, "The New Cordays: Helen Craik and British Representations of Charlotte Corday, 1793-1800 », Adriana Craciun aborde un aspect spécifique de la Révolution, alors qu'un chapitre "Representing Revolution" dans l'ouvrage de Grenby Anti-Jacobin Novel montre comment les descriptions d'événements en France étaient rapidement devenues des formules. Dans son essai, « Rebels Denied a Cause ", Grenby traite en particulier des représentations de la révolte de 1798 en Irlande, événement alors utilisé pour évoquer la possibilité d'une révolution dans un pays plus proche pour des lecteurs britanniques. Au XIX e siècle, la Révolution française était toujours représentée dans les œuvres de fiction; elle procurait une toile de fond exaltante pour les aventures des protagonistes, et était aussi le creuset dans lequel les opinions politiques se formaient. Quelques exemples représentatifs des nombreuses études sur la vie ultérieure de la Révolution française dans les romans sont l'essai de Fred Botting, "Reflections of Excess; Frankenstein, the French Revolution and Monstrosity ", l'essai de David Lodge à propos de Tales of Two Cities de Charles Dickens, "The French Revolution and the Condition of England: Crowds and Power in the Early Victorian Novel ", celui de Kurt Tetzeli von Rosador: "Metaphorical Representations of the French Revolution in Victorian fiction", l'article d'Andrew Sanders: " "The French are always at it": the Impact of the French Revolution on Nineteenth Century English Literature, 1815-1870", et le livre de Doris Kadish, Politicizing Gender: Narrative Strategies in the Aftermath of the French Revolution, qui examine comment les réécritures de la Révolution française par des écrivains français et britanniques du XIX siècle construisirent différemment les rôles des femmes.

La Révolution française et les écrits divers

Tout comme le roman fut rapidement entraîné dans la controverse de la Révolution française, il en advint de même de presque toutes les autres formes d'écrits, qu'ils soient de fiction ou non. Luisa Calè, par exemple, a même recherché quel impact la 
Révolution avait eu sur des livres de botanique («"A Female Band despising Nature's Law": Botany, Gender and Revolution in the 1790s "). Les tracts politiques, tels ceux d'Edmund Burke, de Thomas Paine et de Mary Wollstonecraft, étaient bien sûr des éléments plus évidents dans le débat sur la Révolution française. Ils ont été étudiés de manière exhaustive, que ce soit en termes de contenu politique ou de qualités de forme et de style; les manuels de botanique n'étant pas purement et simplement « de la littérature ", on ne les étudiera pas ici ${ }^{40}$. Toutefois, tout comme les livres de botanique ont une frontière commune avec la littérature pour enfant, qui sera étudiée plus tard, les traités et tracts politiques ont beaucoup en commun avec la littérature populaire. Les opuscules émanant des radicaux dans les années 1790, mais plus particulièrement des anti-jacobins, étaient destinés à faire de la propagande, et ils étaient conçus pour être efficaces précisément parce qu'ils imitaient les publications bon marché qui, des générations durant, avaient été lues par plaisir par les couches les plus pauvres de la société. Une étude récente qui souligne l'impact de la Révolution française sur cette littérature est The Revolution in Popular Literature: Print, Politics and the People, 1790-1860 de Ian Haywood. Il affirme que c'est cette collision entre la culture populaire et la politique des années 1790 qui causa la transformation de la littérature populaire: « d'écrits divers plébéiens » elle devint cette espèce de journalisme et de fiction à grand tirage qu'elle fut à l'époque victorienne.

36 Haywood inclut, bien entendu, un chapitre sur la collection Cheap Repository Tract d'Hannah More, car ce fut l'initiative de More qui tenta le plus ouvertement d'imiter les livres de colportage qui, craignait-elle, vendaient le radicalisme au détail auprès d'un lectorat de masse. Le but était de les remplacer par des textes inoffensifs ou salutaires d'un point de vue politique et moral. Robert Hole («British Counter-Revolutionary Popular Propaganda in the 1790s»), Mitzi Myers («Hannah More's Tracts for the Times ») et Susan Pederson (" Hannah More Meets Simple Simon »), de même que Anne Mellor et Elizabeth Kowaleski-Wallace dont les ouvrages sur More ont déjà été mentionnés, examinent tous Cheap Repository Tracts de manière intéressante. Les pamphlets plus virulents et strictement politiques publiés pour les masses au début des années 1790 par des anti-jacobins aux motivations religieuses moindres ont été moins étudiés. Nombre d'entre eux, élaborés sous les auspices de l'»Association pour la Préservation de la Liberté et de la Propriété » en 1791-92, et également après, ont été reproduits dans la collection en huit volumes Political Writings of the 1790s de Gregory Claeys. Les textes loyalistes sont en général plus « littéraires " que leurs équivalents radicaux, du fait que leurs vues politiques sont exprimées sous une forme plus ou moins romancée ou par des dialogues, comme cela était courant dans la littérature de colportage. Ils portent des titres tels que A Whipper for Levelling Tommy; in Which the Modern Doctrines of the Rights of Man are Properly Stated (1793) ou A New Dialogue between Monsieur Francois and John English on the French Revolution (vers 1793). Mark Philp a examiné leurs stratégies en termes de rhétorique dans son article «Vulgar Conservatism ».

37 De nombreuses satires, elles aussi destinées à être des instruments de propagande au cours de la crise de la Révolution, mais plus franchement «littéraires», firent leur apparition dans les années 1790 . Bien qu'elles aient été peu étudiées, nombre d'entre elles ont été rassemblées par John Strachan dans les cinq volumes de sa collection: British Satire, 1785-1840. On peut y trouver des satires révolutionnaires et conservatrices, telles que, King Chaunticlere; or, The Fate of Tyranny (1793) de John Thelwall ou Daniel Isaac Easton, que l'attaque menée par Richard Polwhele contre les femmes écrivains 
d'inspiration radicale dans: The Unsex'd Females (1798). On peut également trouver des textes politiquement engagés, mais plus ambigus, tels Song, by Mr. Paine (1791) et Ode to Burke (1792) de Peter Pindar. Strachan, avec Graeme Stones, a aussi élaboré une édition annotée de l'abrégé le plus célèbre de satires et parodies politiques de cette époque, The Anti-Jacobin (premier volume de la collection Parodies of the Romantic Age chez Pickering and Chatto). Emily Lorraine De Montluzin, dans The Anti-Jacobins, présente un examen du mensuel le plus ardent, voire le plus polémique, Anti-Jacobin Review, qui, c'est à s'y perdre, prit la suite de l'hebdomadaire Anti-Jacobin. Le journalisme qui apparut de l'autre côté du débat politique est étudié par Brian Rigby dans son essai concernant Analytical Review. Pour Stuart Andrews, le plus grand exploit de la presse anti-jacobine fut de diviser la nation en deux camps opposés : jacobins et loyalistes. Ceci permit au premier ministre, et aux forces du conservatisme plus généralement, de prétendre que chaque Britannique qui ne soutenait pas le gouvernement et sa politique devait être révolutionnaire. L'examen du journalisme britannique dans les années 1790 fait par Andrews dans The British Periodical Press and the French Revolution, montre comment ceci fut réalisé. On peut trouver des compléments d'informations dans un autre livre Pulpits, Politics and Public Order in England de Robert Hole, dans lequel il observe les moyens par lesquels une hégémonie conservatrice s'était établie par les mots écrits - et parlés cette fois-ci par l'intermédiaire de l'Église.

38 Plus surprenant peut-être fut l'impact de la Révolution française sur la littérature enfantine. De nombreux écrits des années 1790 le reconnurent, bien que cette reconnaissance fît généralement partie d'une polémique anti-jacobine. Sarah Trimmer, par exemple, un des auteurs les plus marquants de livres pour enfants, et un de leurs commentateurs, mais également une anti-jacobine convaincue, craignait que la Révolution en France ne fût un élément d'une " conspiration contre le christianisme et tout ordre social » et que les jacobins s'étaient engagés à répandre leurs idées "par l'intermédiaire de livres d'éducation et livres pour enfants " ${ }^{41}$. On se serait attendu à ce qu'elle combatte le feu par le feu, comme tant de romanciers conservateurs l'avaient fait, et qu'elle remplisse ses livres pour enfants de polémiques conservatrices, mais il n'en fut rien. La crainte de politiser l'enfance, que l'on réinventait alors en tant que période d'innocence qui ne devait pas être corrompue par des préoccupations d'adultes, fut semble-t-il trop grande ${ }^{42}$. Même William Godwin, qui écrivit et publia des livres pour enfants dans les années 1800 , se retint de politiser sa production. Les agents du gouvernement restèrent soupçonneux vis-à-vis de son entreprise de publication pour enfant, mais même eux ne purent trouver de preuve qu'il pervertissait la jeunesse par des doctrines radicales ${ }^{43}$.

39 L'article de M.O. Grenby, «Politicising the Nursery: British Children's Literature and the French Revolution ", examine ce conflit entre la nouvelle construction romantique de l'enfance qui se faisait jour et les inquiétudes de plus en plus vives concernant la radicalisation de la littérature enfantine, et étudie également plusieurs textes qui ne manifestaient aucun des scrupules de Trimmer ou de Godwin. Parmi ceux-ci se trouvent des manuels d'histoire qui couvraient de mépris la France postrévolutionnaire et des romans pour enfants ayant pour cadre la Révolution, tels que New Tales of the Castle; or, The Noble Emigrants, a Story of Modern Times (1800) de Mrs. Pilkington. Tout aussi politique était "The Little Hay-Makers ", une des histoires contées dans Select and Entertaining Stories for the Juvenile or Child's Library (vers 1800), qui tentait de montrer que c'était folie que chercher à changer de position dans la hiérarchie sociale. D'une manière générale, ceux qui étaient tout disposés à inclure des 
opinions politiques dans leurs livres pour enfants étaient des auteurs anti-jacobins relativement mineurs. D'autre part, une analyse serrée de textes écrits par certains auteurs qui étaient à la fois radicaux et célèbres peut révéler le contenu politique de leurs livres pour enfants, comme Gary Kelly l'a fait par exemple dans son étude d'Original Stories ${ }^{44}$ de Wollstonecraft.

Pour la plupart des commentateurs, cependant, c'est l'absence de la politique dans la littérature enfantine qui est restée le principal centre d'intérêt. Mary Jackson, dans le chapitre "The Propaganda War in Lilliput » de son livre Engines of Instruction, Mischief, and Magic, considère que de nombreux livres pour enfants ont été politiques, mais son argument d'ensemble est que la crainte de politiser les enfants obligeait les auteurs et les éditeurs à produire davantage de livres fantaisistes qui étaient plus éloignés des réalités de la vie. Dans son essai, "Wordsworth, Fairy Tales, and the Politics of Children's Reading", Alan Richardson fonde son argumentation sur une base plus solide; il suggère que Wordsworth, au cours des années où il fut conservateur, en vint à soutenir que les contes de fées et histoires folkloriques constituaient des lectures naturelles pour les enfants précisément parce qu'elles étaient sans danger, exemptes de politisation (Richardson s'étend sur ce sujet dans Literature, Education and Romanticism).

41 Les histoires et comptines en apparence pour enfants n'étaient pas toujours apolitiques, cependant, ainsi que le montre Andrea Immel dans son article : « Nursery Rhymes in Anti-Jacobin Satire: Baptist Noel Turner's Infant Institutes 1797 ». Les Infant Institutes sont souvent considérés comme les premiers recueils écrits de nombreuses comptines qui étaient, et allaient rester, des éléments de base de culture orale chez les enfants, mais la raison pour laquelle Turner les publiait était complètement politique. Turner, anti-jacobin ardent, imprimait les comptines comme prétexte à ses annotations caustiques dans lesquelles il assimilait les personnages et événements de la comptine aux radicaux britanniques bien connus et aux membres de l'opposition. Ainsi la femme dans "The Old Woman Tossed up in a Basket", par exemple, était quelque " hérésiarque, ou réformateur philosophe » - William Godwin ou Joseph Priestley peutêtre - et le panier symbolisait son parti. Que la femme tente de nettoyer le ciel avec un manche à balai, écrit Turner, est un signe certain de sa folie, de son insolence et de son impiété ${ }^{45}$. Immel révèle que les critiques, et c'est étrange, d'habitude si prompts à saisir les résonnances politiques de toutes sortes de littératures ne semblèrent pas reconnaître le sujet sous-jacent de Turner, éminement politique. Nous pouvons ainsi apprendre deux choses des Infant Institutes, qui à coup sûr sont applicables dans un contexte plus général. D’abord, le bouleversement politique causé par la Révolution française s'est retrouvé dans toutes les formes de littérature sans exception. Ensuite, il arrive parfois que le contenu politique, à nos yeux plus qu'évident dans ces textes, n'ait pas toujours été perçu par ceux qui les ont lus lorsqu'ils ont été publiés pour la première fois. 


\section{NOTES}

1.HAzLITT, Lectures on English Poets (Conférences sur les poètes anglais), 1818, in Complete Works of William Hazlitt, t. V, 161

2.SHELLEY, A philosophical View of Reform (1819), in wHITE (ed.) Political Tracts of Wordsworth, Coleridge and Shelley, p. 227. Le même passage se trouve dans la conclusion de Shelley dans « A Defence of Poetry », 1821; voir p. 206.

3.WORDSWORTH, The Prelude, livre XI, 1.108

4.De QUINCEY, « William Wordsworth », in Collected Writings of Thomas De Quincey, II, 273-274

5.Abrams a ultérieurement tenté d'expliquer que sa thèse ne devait en aucun cas se réduire à une simple théorie sur les origines du romantisme et sa définition: voir « Revolutionary Romanticism 1790-1990 » d'Abrams.

6.D'autres écrits significatifs de Butler, qui dans l'ensemble concernent ce sujet, comprennent : « Plotting the Revolution : The Political Narratives of Romantic Poetry and Criticism » et « Revolving in Deep Time: The French Revolution as Narrative». Voir Rousseau, Robespierre and English Romanticism de Gregory DART pour une étude nouvelle de l'impact de la pensée du siècle des Lumières sur les écrivains britanniques, qui développe les idées de Deane en soulignant l'importance de la rhétorique révolutionnaire pour transmettre cette pensée.

7.Mee revint sur ces idées dans une certaine mesure, de même qu'il élargit son champ de vision pour inclure beaucoup d'autres écrivains romantiques dans son livre ultérieur Romanticism, Enthusiasm, and Regulation.

8.Ainsi que l'écrit Mee : « Erdman a tendance à identifier la dimension politique de la poésie et de l'œuvre graphique de Blake à la représentation d'événements historiques. Cette tendance signifie que, à son tout début, la critique historiciste de Blake sousestimait la signification révolutionnaire de la dimension formelle de ses œuvres. La pratique de Blake en ce qui concerne la forme a autant de signification politique que sa représentation de révoltes historiques dans des poèmes tels que The French Revolution et America » (pp. 1-2).

9.MAKDISI, William Blake and the Impossible History of the 1790s, p. 3.

10.LeVInson, Wordsworth's Great Period Poems, pp. 93-94 et 98-99.

11.LIU, Poetics and Politics, p. 1.

12.HANLEY et SELDEN, «Préface » à Revolution and English Romanticism, p XIV.

13.Sur la question politique dans Prelude de Wordsworth, voir également pp. 248-285 in Representations of Revolution de Ronald PAULSON, et ce qu'écrit Geraldine FRIEDMAN dans le chapitre « The Letter and the Spirit of the Law: Wordsworth's Restagings of the French Revolution in The Prelude » de son ouvrage The Insistence of History, pp. 64-90.

14.Roe Wordsworth and Coleridge, p. 265. Voir également de Peter KITSON, «Coleridge, the French Revolution and The Ancient Mariner ", de Morton D. PALEY « "These Promised Years": Coleridge's "Religious Musings" and the Millenarianism of the 1790s ", et de Robert SAYRE « The Young Coleridge: Romantic Utopianism and the French Revolution».

15.Jon MEE, « Mopping Up Split Religion: The Problem of Enthusiasm », paragraphe 11. 
16.WAKE, « Indirect Dissent: “Landscaping” Moral Agency in Amelia Alderson Opie’s Poems of the 1790s », p. 262.

17.cox, "The French Revolution in the English Theater », p. 34

18.JOHNSON and NICHOLES, « Transitory Actions, Men Betrayed: The French Revolution in the English Revolution in Romantic Drama », pp. 93 et 76.

19.cox, « The French Revolution in the English Theater », p. 42.

20.TAYLOR, French Revolution and the London Stage, p. 99.

21.Taylor fait bien une brève analyse des sens politiques multiples de Pizarro dans The French Revolution and the London Stage, pp. 172-176.

22.Paula R. BACKSCHEIDER, Spectacular Politics, p. 149.

23.Ibid., pp. 226, 232 et 233.

24.Une autre analyse du programme radical de Baillie se trouve dans l'essai de Terence HOAGWOOD « Elizabeth Inchbald, Joanna Baillie , and Revolutionary Representation in the "Romantic" Period».

25.GREEN, « Mr Harmony and the Events of January 1793 », p. 62. En fait, au moins par deux fois, à Brighton en 1793 et Portsmouth en 1795, les représentations de cette pièce furent accueillies par de violentes bagarres dans le théâtre.

26.FERRISS, « Reflection in a "Many-Sided Mirror": Shelley's The Cenci Through the Post-Revolutionary Prism », p. 142.

27.cox, « The French Revolution in the English Theater », p. 33.

28. La tendance à voir toute la politique comme relevant du théâtre est devenue si répandue que cela a incité Michael Simpson à critiquer cette «tendance dans le nouvel historicisme » qui applique « la notion de "théâtre" si largement et métaphoriquement que cela détourne l'attention critique de l'institution historique du théâtre ». SIMPSON, Closet Performances, p. 14.

29.Voir de GRENBY « Politicised Fiction in Britain 1790-1810 » pour une une liste complète annotée des romans qui ont pris part à la « guerre des idées ».

30.wooD, Modes of Discipline, p. 36. Voir aussi de wooD, « Bachelors and "Old Maids": Antirevolutionary British Women Writers and Narrative Authority after the French Revolution ».

31.Sur le même sujet, voir « Charlotte Smith's Desmond. The epistolary novel as Ideological Argument » et de Mary A. FAVRET, Romantic Correspondence: Women, Politics and the Fiction of Letters.

32. WATT, Contesting the Gothic, pp. 7-8. Le second chapitre de Watt traite en particulier de « The Loyalist Gothic Romance ».

33. WALKER, The Vagabond, I, VI.

34.JOHnson, Jane Austen: Women, Politics and the Novel, p. XXI.

35.Eighteenth-Century Fiction, 13 (2000), pp. 98-99

36.TY, Unsexed Revolutionaries, p. 28. Voir aussi « Female Philosophy Refunctioned:

Elizabeth Hamilton's Parodic Novel » de Ty.

37." Toutes deux, poursuit Myers, faisaient partie d'une campagne de la bourgeoisie, de plus grande ampleur et finalement couronnée de succès pour réhabiliter une culture dégénérée, utilisant de la propagande pour une vie familiale éclairée et la réforme sociale », « Reform or Ruin », pp. 211-212.

38. MELLOR, Mothers of the Nation, p. 142. Des conclusions identiques se trouvent dans The Domestic Revolution de BANNET, voir également « Hannah More’s Counter-Revolutionary Feminism » de SUTHERLAND. 
39.Churchill cité dans Jane Austen and the French Revolution de Roberts, p. 5.

40.Quelques unes des meilleurs études sur la langue de la politique dans ces publications sont, d'Olivia SMITH, The Politics of Language et de Tom FURNISS, Edmund Burke's Aesthetic Ideology: Language, Gender and Political Economy in Revolution, et " Rhetoric in Revolution: The Role of Language in Paine's Critique of Burke ». Le meilleur résumé de l'engagement de Wollstonecraft vis-à-vis de la Révolution est « Mary Wollstonecraft and the French Revolution » de FurNISS.

41.TRIMMER, Guardian of Education, I (1802), 2.

42.D'après Carl F. Fisher, cependant, Hannah More écrivit bien des livres pour enfants anti-jacobins, mais il parvient à cette conclusion après avoir considéré que Cheap Repository Tracts de More avaient été destinés aux enfants: « Politicising Childhood: Hannah More, The Riot, and Children's Books in an Age of Revolution ». Pour en savoir davantage sur les alignements politiques de Trimmer, voir de M.O. GRENBY, «"A Conservative Woman Doing Radical Things": Sarah Trimmer and the Guardian of Education » et d'Andrea IMMEL, Revolutionary Reviewing: Sarah Trimmer's Guardian of Education and the cultural politics of juvenile literature.

43.Pour une description de l'entreprise de publications pour enfants de Godwin, voir « Godwin as Children's Bookseller » de William St. CLAIR, «Childhood and Children's Literature: The Case of M.J. Godwin and Co., 1805-1825 » de Margaret KINNELL et «"Mister Godwin" and His "Interesting Little Books, Adorned with Beautiful Copper Plates" » de Brian ALDERSON.

44.KELLY, Revolutionary Feminism, pp. 58-67.

45.IMMEL, « Nursery Rhymes in Anti-Jacobin Satire », p. 41.

\section{RÉSUMÉS}

Il est largement admis que la Révolution française a eu un impact énorme sur la littérature anglaise et que pratiquement tous les poètes, romanciers et auteurs dramatiques de cette époque ont écrit des textes influencés par les principes et par les remarquables événements français. Cet article se penche sur les travaux récents consacrés aux écrivains de fiction que la Révolution française a enthousiasmés, à ceux qu'elle a effrayés ou horrifiés, et à ceux qui, après l'avoir saluée, l'ont ensuite critiquée.

The French Revolution and English Literature. It is widely recognised that the French Revolution had an enormous impact on English literature and that almost all contemporary poets, novelists and playwrights wrote works directly influenced by French principles and by the remarkable events in France. This essay looks in particular at recent studies of those writers of imaginative literature who were excited and attracted by the French Revolution, those who were frightened and appalled by developments in France, and those who initially welcomed the French Revolution but later became its critics. 
INDEX

Mots-clés : théâtre, radicaux, conservateurs, poésie, roman

\section{AUTEUR}

\section{MATTHEW O. GRENBY}

University of Newcastle, Department of English Literature, Newcastle upon Tyne, NE1 7RU, Royaume-Uni 